\title{
Assessing the impact of a new central venous access device training program for nurses: A Quasi-Experimental Evaluation study
}

Burt, W

http://hdl.handle.net/10026.1/17501

\subsection{7/1757177420982041}

Journal of Infection Prevention

SAGE Publications

All content in PEARL is protected by copyright law. Author manuscripts are made available in accordance with publisher policies. Please cite only the published version using the details provided on the item record or document. In the absence of an open licence (e.g. Creative Commons), permissions for further reuse of content should be sought from the publisher or author. 
ASSESSING THE IMPACT OF A NEW CENTRAL VENOUS ACCESS DEVICE TRAINING PROGAM FOR NURSES: A Quasi-Experimental Evaluation study.

Funding: This research did not receive any specific grant from funding agencies in the public, commercial, or not-for-profit sectors.

Word Count: 3961 


\section{Abstract}

Background: Central Venous Access devices (CVAD) are widely used for both long and short-term purposes within healthcare and are suitable for both hospital and community management. Training is essential in the prevention of complications such as infection. Objectives: To assess the impact of a new standardised education program on clinical practice and patient care. The new education program was introduced to all RN's working in one care group within an acute healthcare Trust with the aim to improve knowledge and skills and reduce CVAD related complications.

Methods: This retrospective Quasi-Experimental evaluation study analyses the impact of the program on direct patient care. Secondary data sources such as infection incidence rates and CVAD clinical audits were used to identify and measure the relationship between staff confidence, infection incidence and care audit results. Data spanning a 2 year period was used to capture an accurate representation of the patient group.

Findings/Results: Improvements in audited care elements and a reduction of infection incidences were evident during and after implementation of the education program. This was reflective of the self-reported increased confidence and knowledge and skill acquisition from staff who attended the program. Discussion: Recommendations have been made including a review of the education content to target all key elements and promotion of an end goal with regular feedback to staff reinforcing the importance. The challenge of using secondary data sources also highlighted the need for quality improvements in the current care audit process.

Key words:

Education

Evaluation

Central venous Access Device

Nursing

Infection 


\section{Background}

Central Venous Access devices (CVAD) are widely used for both long and short-term purposes within healthcare and are suitable for both hospital and community management. These devices carry with them significant risk to the patient including the potential for infection. NHSN (2012) report Central Line Associated Blood Stream Infection (CLABSI) rates as 2.2 to 2.6 per 1,000 catheter days for paediatric, cardiothoracic, medical, or medical/surgical Intensive Care Units (ITU). This potentially fatal line related infection, along with numerous other complications are widely recognised ( $O^{\prime}$ Grady et al., 2011, Conley, 2016, Gabriel, 2020). CLABSI can be defined as a blood stream infection that arises from a CVAD when there is no other source of infection identified, (Nicholson, 2018). Insertion, management and removal of CVAD, best care practices are documented through internationally recognised guidelines such as the recommendations published in the epic2 report, (Pratt, et al., 2007), which was later updated to epic3 (Loveday, et al., 2014). The revision included new recommendations around vascular access care for example, education of staff in areas such as, Asepsis, manufacturer's advice related to indwelling medical devices, cleansing and dressing selection. NICE (2015) also published guidance around dressing selection in support of epic3. Individual organisations have developed policy documents and training programmes to encourage best evidence-based practice. In the development of local policies and guidelines, consideration is given to cost implications, resource availability and current education practice, which explains the variations across organisations.

Despite a nationally recommended standardised approach to the management of CVAD, infections still prevail. This is attributed to numerous factors such as: patient condition; the knowledge and skills of those delivering care, and adherence to policy and guidelines. Studies have identified a knowledge and skills deficit amongst professionals inserting and managing CVADS (Ullman et al., 2014; Paolucci and Albert, 2011). One influencing factor may be the way in which training and 
assessment is conducted which is variable across organisations (Kelly, et al 2019). This paper evaluates the impact of a new standardised education program aimed at improving the knowledge and skills of nursing staff in relation to CVAD management.

A literature search spanning 2010-2019 identified few studies which directly focused on the evaluation of CVAD education (Holt et al., 2010; Mathers, 2011; Raup et al., 2013; Kelly et al., 2015; Hainey et al., 2017). Although it could be argued that clinical studies from the previous decade may be too old to reflect current practice it was decided as the focus was on education to expand the search back to the year 2000 which identified 3 further relevant papers (Coppersmith et al., 2002; Warren et al., 2004; Ngo \& Murphy, 2005). Coppersmith et al., (2002) and Warren, (2004) focused on the reduction of infection rates as the indication of a successful training program. Both studies include a purposive sample of staff on one critical care unit and used a pre-and post-intervention methodology. Both studies declared a reduction in infection incidences per 1000 catheter days. Ngo and Murphy (2005), Holt et al., (2010) and Mathers, (2011) focused on occlusion rates as the measurable outcome and reported that a reduction was achieved. Pre- and post-testing was also used to evaluate the education program and improved knowledge scores are reported. Holt et al., (2010) and Mathers (2011) both included primary data from direct observation of practice. However, there were some limitations to this element such as some being observed in a simulated environment and concepts such as the 'observer effect' and 'Hawthorne effect' would have been difficult to eliminate (McCambridge, 2014, Hurley et al., 2015).

The later studies by Raup et al., (2013), Kelly et al., (2015) \& Hainey et al., (2017) all aimed to reduce infection incidences. Raup et al., (2013) included all staff and reported to have achieved a target infection rate of zero. The focus of this education was to foster a culture of change so the education was supported with continuous reinforced information and publications to maintain focus of the CVAD care among staff. Kelly et al., (2015) followed by Hainey et al., (2017) began with a face to face training initiative supported by a University and followed up with a blended learning approach by 
adding E learning. Although there are limitations in regards to the population who participated or completed training, this blended approach was reported favourably by staff and a reduction in infection rates across the healthcare organisation is reported.

Few of the studies identified, have attempted to analyse more than one or two measurable outcomes, therefore, the transferability and evidence of direct association with any clinical impact are limited. A common theme has been to evaluate staff knowledge and confidence with the skills required for CVAD management along with infection incidences. Standing alone, this approach is insufficient to directly link the education to a reduction in CLABSI and it could be argued that pre and post knowledge testing should have a predictable positive outcome.

\section{Method}

This study focuses on a mandatory standardised education program which was introduced in 2015 to a specific care group within an acute Healthcare Trust. Unlike the previous CVAD training, the program was designed and delivered by the clinical nurse education team. A retrospective approach was taken using secondary data sources in an attempt to reduce researcher bias in the data collection process. Secondary data sources refers to data which has been collected for a different purpose to the current study, but can still prove to be useful for further review purposes. (Allen, 2017)

The delivery of the educational intervention was monthly between October 2015 and September 2016, capturing 112 registered nurses which represented $97 \%$ of the total staff pool within the specific clinical care group. The group comprised of 2 surgical wards (labelled ward 1 and ward 2 for the purpose of this evaluation), a surgical Intensive Care Unit and surgical High Dependency Unit.

To achieve a standardised approach the training was delivered and all competency assessments were completed by the same 2 clinical educators. Inter-rater reliability was considered by the education team to ensure that all competencies were completed with the same expected standards. 
For this in-house assessment a simple 'percentage agreement' level of reliability was considered sufficient (Downing, 2004).

The program included, didactic lecture with interactive opportunities, practical demonstrations and 2 practical task simulation activities with questioning to enable competency assessment.

The 3 data sets used were:

1. A staff education feedback tool

2. A review of CVAD infection incident rates

3. $2 \times$ CVAD management audits.

The re-use of existing data to address new research questions is established within the social sciences, this was not an attempt to recreate any original research, but rather to contextualise new perspectives or themes within previously collated data sets (Hammersley, 2010). Utilising existing data in this way can therefore potentially have positive implications on resources and knowledge. Data triangulation also contributes to the understanding and validity of the impact findings (Altrichter et al., 2008). The use of one or two measurable outcomes only, for example infection rates and/or knowledge tests, was found to be a limiting factor in the fore mentioned studies.

\section{Staff education feedback Questionnaire}

A feedback questionnaire was written by the education team to evaluate success and inform future programme design and delivery. This was completed anonymously by participants after attendance. The format used was a retrospective pre-post design using Kirkpatrick's (2006) evaluation process. The benefit of a retrospective pre-test or feedback tool is suggested by Allen and Nimon (2007) as a way to avoid response shift bias which can occur with pre-and post-test methods. Using reflective questioning, attendees were asked to assess their own progress to provide a more accurate description of their own development. 


\section{CVAD Infection Rates}

The second outcome measure was the incidence of CLABSI. This information is routinely collected and reported by the hospital infection control team in collaboration with the microbiology department. The data was provided by the data analyst for the infection control team in the form of absolute numbers. The criteria for identification of a CVAD related bacteraemia is set by the infection control and microbiology team.

\section{CVAD management audits}

The next 2 sets of secondary data analysed were the results of the CVAD audit tools completed monthly from all the clinical areas. There were 2 clinical audits included.

1. Acute Care Team (ACT) CVAD audit.

2. Trust adapted National Saving Lives data collection tool (DOH, 2008).

\section{Results.}

The data sets were analysed using basic frequency, describing the results in absolute numbers, percentages and proportions (Parahoo, 2014). The data sets were collated manually by tally of frequency and presented in a diagrammatic style to give a sense of proportion.

The feedback questionnaire had an $86 \%$ response rate. As expected declaration of prior learning indicated a mixed experience of CVAD training prior to this program. Responses indicated the acquisition of new knowledge and skills during the training and a significant increase in confidence in CVAD management was reported. $45 \%$ of the staff stated they felt more confident in sharing their knowledge and skills after the training which indicates an intention to disseminate knowledge and $82 \%$ of the respondents stated that they would make a change to their own clinical practice as a result of the training indicating the intent to transfer knowledge and skills to practice. 
The CVAD infection incidences data was provided in the form of absolute numbers per month for each area. The 2 critical care areas are reported as one by the infection control team. Diagnosis of CLABSI is made by the Trust infection control team and microbiology department in line with the current organisational policy. The process involves systemic and line blood culture samples, removal of line and tip culture, as well as a site swab if indicated.

The most significant change was seen across the critical care areas. From 6 months prior to the education programme commencing to the 6 months after delivery there was an $83 \%$ reduction in CLABSI across the critical care areas, (Figure 1) There were no changes to policy, speciality, equipment or patient dependency changes beyond usual variability within the unit. There was also a reduction of infections on ward 2, from year 1 to year 2 . Although infection incidences are low in numbers, in the first instance it must be recognised that this is the expectation, high infection incidences carry significant risk to the patient and cost to healthcare organisation therefore these numbers are expected to run low, consequently even a small increase or decrease is considered significant and the aim is to achieve a 0 incidence rate.

Audit 1 - ACT CVAD Audit.

Unfortunately there were gaps in the data provided by the ACT. This was due to the collector being absent for a 6 month period. Despite the missing data recurring themes were identified. 154 lines were audited over the initial 18 month period. The care elements included can be found in Table 1. The most common theme was dressing errors. $32 \%$ of the lines observed dressings were either not fully intact, soiled or not dated. Dressing errors reduced by $9 \%$ during the implementation phase of the programme across the clinical area. 
The second theme identified is the presence of the correct needleless connector or 3-way tap device attached the CVAD. $8 \%$ of lines had incorrect needless connectors in place. This reduced to 0 in the last half of the implementation period and is indicative of an improvement in practice.

Audit 2 - TRUST SAVING LIVES Audit.

On accessing this audit data is was discovered that the questions which were used pre-education were expanded from 6 to 13 during the implementation phase. Therefore, the key 6 questions which present data from before, during and after education were examined first.

Analysis of this data identified discrepancies in auditor understanding and interpretation. Responses appeared contradictory and inappropriate in places. For example, the answer was often yes to the site being observed at least daily for signs of infection but no to the recording of the Central Catheter Assessment Tool (CCAT) score. This raises the question, how did the auditor know it had been observed if the CCAT score had not been documented? The audit suggests that an observation of practice should take place for it to be completed fully and accurately however responses indicated that this was not always the case. Despite these findings there did appear to be some improvement in simple care elements that mirrored the ACT audit. The audit questions can be seen in table 2 .

\section{Discussion.}

Individually each set of data showed varying degrees of practice improvement. Whilst previous studies (e.g. Raup et al., 2013; Kelly et al., 2015; Hainey et al., 2017), have successfully shown some improvement, they have limited their results to only one or two measured outcomes.

The analysis of multiple data sources makes this study unique to previous CVAD training evaluation studies and minimises the influence of researcher bias. Each data set looks at a different aspect of CVAD management, including, education programme evaluation, care audits and infection incidences. 
In this evaluation those who attended the CVAD education programme were directly responsible for the care given to the patients with a CVAD within the clinical areas. Although infection data was as expected, in small numbers, a reduction in infection rates is shown post education. This reduction was not as significant as that shown by Raup et al., (2013), who reported to have achieved an infection rate of Zero post implementation. However, Raup et al.'s, (2013) educational programme was continually reinforced with monthly reports to staff which is an important consideration in terms of knowledge retention.

The studies reviewed have demonstrated a number of strategies to support education initiatives. These have included promotional information, adaptation of the clinical environment, the use of secondary education sources and scheduled reinforcement. The studies which included these elements appear to have had the most significant results. This continuous re-enforcement approach has been shown to improve learning and continue knowledge and skills acquisition through repeated exposure and reminders of key principles (Haggmann-Laitila et al., 2016).

The CVAD management audits in stage 3 showed an improvement in many care elements which had been included in the education programme. Dressings were correct and dated, the correct needless connectors were being used and there was an improvement in the use of infusion lines. Had a continuous reinforcement plan been utilised following the training, these results may have been more significant.

Reviewing the education program prior to further delivery will ensure it meets the needs of the learners and is comprehensive at the planning stage. A focus on documentation is clearly required as this care element showed no practice improvement across both audits. Utilising a pre-analysis method to target education more specifically to problem areas is more likely to lead to improved practice, (Donaldson et al., 2017)

\section{Limitations.}


The use of secondary data sources led to some incomplete data from one audit tool due to auditor absence and some auditor bias from another which appears to be from lack of education and training around the process of audit and interpretation of the questions. Bias from a systematic error cannot be controlled or eliminated and no analytical approach can fully provide unbiased estimates for the missing data which may have impacted the validity of results (Enders, 2013). As in this case the missing data from observational audits cannot be added or calculated retrospectively as it is reliant on direct observation in clinical practice.

\section{Conclusion}

In summary, all 3 data sets have indicated some improvement in CVAD management across the Care group who received the new education programme. The reduction in infection incidences and the improved care elements are reflective of the increased knowledge, skills and self-reported confidence of the attendees which suggests there was direct transfer of knowledge and skills into patient care.

Infection prevention and control strategies are essential for patient safety within healthcare organisations and one element of this is staff education. However, this project has identified that although educational programmes, can be delivered and received effectively, it is important to consider how to measure success through patient and practice outcomes to ensure the knowledge and skills are transferred to patient care.

\section{Recommendations.}

This evaluation study identified factors which require improvement both in the specific education program and the data sets which were examined. The education program has since been reviewed and updated.

- A positive reinforcement plan to maintain interest and engagement from staff after training. 
- A blended learning approach. Including an E learning update package to be completed in between face to face teaching focusing on organisational CVAD care policy and guidelines.

- An audit review by the owners to ensure data that is captured is accurate and complete.

\section{Acknowledgement:}

Rachel Allen, Cardiothoracic Clinical Educator for provision and delivery of teaching resources for the education program.

\section{The Authors declare that there is no conflict of interest}

\section{Ethical approval}

Whilst the data was not collected for the purpose of this research, ethical approval was sought retrospectively via the local University as this study was completed as a masters in clinical education dissertation. Authorisation was also obtained via the Trust quality standards team to access the necessary secondary data sets for analysis. No identifiable patient information was accessed for the purpose of this study. 


\section{REFERENCES}

1. Allen, J., M., Nimon, K., (2007). Retrospective Pre-test: A Practical Technique for Professional Development Evaluation. Journal of Industrial Teacher Education, 44(3), pp. 27-42.

2. Allen, M., (2017) Secondary Data. The Sage Encyclopaedia of Communication research methods. Available at: http://dx.doi.org/10.4135/9781483381411.n557. (Accessed 12/09/20)

3. Altrichter, H., Feldman, A., Posch, P., Somekh, B., (2008). Teachers investigate their work: An introduction to action research across the professions. 2nd ed. London: Routledge.

4. Conley, S., B., (2016) Central Line-Associated Bloodstream Infection prevention: Standardizing practice Focused on Evidence-Based Guidelines. Clinical Journal of Oncology Nursing. Vol.20(1), pp. 23-26.

5. Coppersmith, C., M., Rebmann, T., L., Zack, J., E., (2002). Effect of an education program on decreasing catheter-related bloodstream infections in the surgical intensive care unit. Critical Care Medicine, Issue 30, pp. 59-64.

6. Department of Health, (2008). Clean, Safe Care. Reducing Infections Saving Lives, s.I.: Department of Health.

7. Donaldson, S., R., Harding, A., M., Taylor, S., E., Valley, H., Greene, S., L., (2017). Evaluation of a targeted prescriber education intervention on emergency department discharge oxycodone prescribing. Emergency Medicine Australasia, Issue May 2017, pp. 1-7.

8. Downing, S., M., (2004). Reliability: on reproducibility of assessment data. Medical Education, 38(9), pp. 1006-1012.

9. Enders, C., K., (2013). Dealing with missing data in developmental research. Child Development Perspectives, 7(1), pp. 27-31.

10. Gabriel, J., A., (2020) Catheter lock solutions to prevent CVAD-related infection. British Journal of Nursing. Vol. 29(3), pp S25-S29.

11. Haggman-Laitilla, A., Mattilla, L., R., Melender, H., L., (2016). Educational interventions on evidence based nursing in clinical practice: A systematic review with qualitative analysis. Nurse Education Today, Volume 43, pp. 50-59.

12. Hainey, K., Green, A., Kelly, L., J., (2017). A blended learning approach to teaching CVAD care and maintenance. British Journal of Nursing, 26(2), pp. S4-S12.

13. Hammersley, M. (2010). Can we re-use qualitative data via secondary analysis? Notes on some terminological and substantive issues. Sociological Research Online, 15(1), 5. 
14. Holt, D., M., Lewis, C., Kimpel, K., Sloan, C., Aguda, C., (2010). The Effects of Focused Nurse Education on 3F Groshong PICC Occlusion Rates: The experience of one tertiary paediatric care facility. JAVA, 15(4), pp. 213-221.

15. Hurley, K., F., Giffin, N., A., Stewart, S., A., Bullock, G., B., (2015). Probing the effect of OSCE checklist length on inter- observer reliability and observer accuracy. Medical Education Online, Volume 20.

16. Kelly, L., J., Green, A., Hainey, K., (2015). Implementing a new teaching and learning strategy for CVAD care. British Journal of Nursing (IV Supplement), 24(8), pp. S4-S12.

17. Kelly, L., J., Snowden, A., Paterson, R., Campbell, K., (2019) Health professionals' lack of knowledge of central venous access devices: the impact on patients. British Journal of Nursing. Vol. 28(14). pp S4-S14

18. Kirkpatrick, D., L., Kirkpatrick, J., D., (2006). Evaluation training programs: The four levels. 3rd ed. San Francisco:Berrettkoehler Publishers Inc.

19. Loveday, H., P., Wilson, J., A., Pratt, R., J., Golsorkhi, M., Tingle, A., Bak, A., Browne, J., Prieto, J., Wilcox, M., (2014) epic3: National Evidence-Based Guidelines for Preventing HealthcareAssociated Infections in NHS Hospitals in England. Journal of Hospital Infection. 86S1, S1S70.

20. Mathers, D., (2011). Evidence-based Practice: Improving Outcomes for Patients with a Central Venous Access Device. Journal of Association of Vascular Access (JAVA), 16(2), pp. 64-72.

21. McCambridge, J., Witton, J., Elbourne, D., R., (2014). Systematic Review of the Hawthorne effect: New concepts are needed to study research participation effects. Journal of Clinical Epidemiology, Issue 67, pp. 267-277.

22. National Healthcare Safety Network, (2012). US Centres for Disease Control and Prevention. [Online] Available at: www.cdc.gov/nhsn/PDFs/pscManual/4PSC [Accessed January 2015].

23. National Institute for Health and Care Excellence (NICE), (2015). The 3M Tegaderm CHG IV securement dressing for central venous and arterial catheter insertion sites. [Online] Available at: https://www.nice.org.uk/guidance/mtg25/chapter/1-Recommendations [Accessed January 2016]

24. Ngo, A., Murphy, S., (2005). A Theory-based Intervention to Improve Nurses Knowledge, Self-efficacy, and Skills to reduce PICC Occlusion. Journal of Infusion Nursing, 28(3), pp. 173181.

25. Nicholson, J., (2018) Royal College of Nursing's Standards for Infusion Therapy: an overview. British Journal of Nursing. Vol. 27, (2)

26. O'Grady, N., P., Alexander, M., Burns, L., A., (2011). Guidelines for the prevention of intravascular catheter-related bloodstream infection. [Online] Available at: http://tinyurl.com [Accessed 1 September 2016].

27. Paolucci, H., Albert, N., (2011). RN Knowledge of vascular access devices management. Journal of Association of Vascular Access, Issue 16, pp. 221-225.

28. Parahoo, K., (2014). Nursing Research Principles, Process and Issues. 2nd ed. Basingstoke: Palgrave Macmillan.

29. Pratt, R., J., Pellowe, C., M., Wilson, J., A., Loveday, H., P., Harper, P., J., Jones, S., R., McDougall, C., Wilcox, M., H., (2007). epic2: National evidence-based guidelines for preventing healthcare-associated infections in NHS hospitals in England. Journal of Hospital Infection, 65(Supplement 1), pp. 1-64.

30. Raup, G., H., Putnam, J., (2013). Can an education program reduce CLABSI? Nursing Management, 44(5), pp. 20-22.

31. Ullman, A., J., Long, D., A., Rickard, C., M., (2014). Prevention of central venous catheter infections: A survey of paediatric ICU nurses\&\#39; knowledge and practice. Nurse Education Today, Issue 34, pp. 202-207. 
32. Warren, D., K., Zack, J., E., Mayfield, J., L., (2004). The effect of an education program on the incidence of central venous catheter-associated bloodstream infection in a medical ICU. CHEST, Issue 126, pp. 1612-1618. 